\title{
An experimental GPR detection study of environmentally-influenced structural defects in hydraulic engineering
}

\author{
DONGJIAN ZHENG ${ }^{1,2,3}$, JIANCHUN QIU $^{1,2,3, * \mathbb{D}}, \mathrm{CHAO} \mathrm{WEI}^{4}, \mathrm{LIN} \mathrm{CHENG}^{5}$ and KAI ZHU ${ }^{3}$ \\ ${ }^{1}$ State Key Laboratory of Hydrology-Water Resources and Hydraulic Engineering, Hohai University, \\ Nanjing 210098, China \\ ${ }^{2}$ National Engineering Research Center of Water Resources Efficient Utilization and Engineering Safety, Hohai \\ Universty, Nanjing 210098, China \\ ${ }^{3}$ College of Water-Conservancy and Hydropower, Hohai University, Nanjing 210098, China \\ ${ }^{4}$ Nuclear and Radiation Safety Center, Beijing 100082, China \\ ${ }^{5}$ State Key Laboratory Base of Eco-Hydraulic Engineering in Arid Area, Xi' an University of Technology, \\ Xi'an 710048, China \\ e-mail: 121267184@qq.com
}

MS received 6 July 2016; revised 22 June 2018; accepted 24 September 2018; published online 20 November 2018

\begin{abstract}
Structural defects such as separation between concrete slab and foundation, and structural voids often occur in hydraulic engineering, which threatens the safety of hydraulic engineering. As the size and internal material of the hydraulic engineering can be queried, ground penetration radar (GPR) detection has the advantage to detect these defects when compared with other nondestructive detection methods. At the same time, when GPR detection is applied to defect these structural defects in hydraulic engineering, complex environmental factors including the uneven structural surface, clutter interference, water reflection, etc. have to be taken into account. In this work, two experimental models are designed to represent two different types of hydraulic structures and the structural defects including separation between concrete slab and its bottom material and void or hole defects are simulated on the two test models, respectively. Through the GPR detection on the two experimental models, the effects of the three environmental factors on the radar images and the signatures of the radar images under the influence of the three environmental factors are studied. Then, different image processing methods are adopted to reduce the influence of the three different environmental factors, and the effects of these methods are verified using the radar images obtained from the experiments. Finally, the GPR detection on a practical hydraulic engineering influenced by the environmental factors and the image processing methods are investigated, which successfully verify the experimental investigation results. It is expected that this study would provide significant technology support for structural defects detection in hydraulic engineering.
\end{abstract}

Keywords. Ground penetrating radar; hydraulic engineering; separation; void; environmental effects; signal processing.

\section{Introduction}

Hydraulic engineering involves environments with characteristically complex hydrology, geology and climate. Due to the influence of water pressure, seepage, earthquake, temperature variation, material ageing and other factors, structural defects such as separation between concrete slab and foundation and structural voids occur in hydraulic engineering, which threatens the safety of hydraulic engineering and may even result in heavy economic, life and property losses.

The separation between concrete slab and its bottom material and voids are two common defects in hydraulic

*For correspondence engineering, and these defects are difficult to detect through field inspection due to being under the ground and not visible. On-site detecting has the advantage of providing a real-time understanding of the structural safety state. However, the structure and environmental factors of hydraulic engineering concerning separation and structural voids are rather complex. Some technologies such as acoustic emission (AE) [1] and vibration tests [2-4] are usually difficult and unsuitable for finding these defects. Since hydraulic engineering are often man-made, the size and internal material could be investigated through design data or other related data. GPR is a detection method [5] used for determining the internal distribution of a medium through high frequency electromagnetic waves and has an advantage in being able to detect these hidden defects, 
which has been widely applied in many fields [6-13]. Compared with other engineering structures, the complex hydrology, geology and climate environment involved with hydraulic structures greatly affect the GPR detection results, which makes it difficult to identify the signatures of structural defects based on radar images. Moreover, studies of GPR detection on structural defects in hydraulic engineering under the influence of environmental effects are few. The uneven ground surface, clutter disturbances and the interference of water are three common environmental factors that affect the final detection results. The separation between a concrete slab and its bottom material and structural voids are two common structural defects in hydraulic engineering. Therefore, before practical implementation of GPR detection in hydraulic engineering, it is of great significance to conduct experimental research on the impact of these three environmental factors on GPR penetration results and methods that can be used to reduce the impact.

In this paper, we describe two common defects and three environmental factors that may affect the GPR penetration data. Then, two experimental models are designed to represent two different types of hydraulic structures, in which the two types of structural defects and three environmental factors are simulated. According to the practical GPR detection result of the two models, different image signatures corresponding to different environmental factors are obtained, which aid image identification in hydraulic engineering. Some image processing methods are introduced to weaken the effects of environmental factors on radar images, and the effects of these measures are verified using the GPR detection profiles obtained through the experiments. By using the GPR detection of a sluice as an example, the image feature and processed measures under the influence of the three environmental factors are verified with the experimental research.

\section{Two common defects and three environmental factors}

The separation between a concrete slab and its bottom material is a common defect in hydraulic engineering. The possible reasons for this include uncoordinated structural deformation and seepage failure. Due to the great difference between a concrete slab elastic modulus and the foundation materials, the deformation of the concrete slab and base materials is uncoordinated when external loads are imposed on the structure, and thus, separation may appear between the concrete slab and its bottom material. A typical example of this is the separation between a concrete slab and the cushion layer in an embankment, which is mainly induced by the construction of a neighbouring embankment body (see figure 1a). Another possible reason for the appearance of a separation is that a seepage failure occurred in the foundation material. If the seepage prevention ability of a foundation is poor or there are cracks present, the seepage flow will erode the concrete structures by dissolving material out of the foundation. Then, the concrete structure loosens due to strength loss of the concrete material. With the leakage flow, soils in the foundation will be gradually brought out, forming small water or mud filled gaps between the concrete slab and foundation. Consequently, the concrete slab will separate from the foundation in these gapped zones. These small gaps often appear on the bottom of sluice floors and would result in voids in the foundation if the leakage was not treated, as seen in figure $1 \mathrm{~b}$. Voids or holes in the structure including seepage holes and animal caves are additional common structural defects encountered in the hydraulic engineering. In addition to the aforementioned voids in sluice foundations caused by seepage failures, voids also occur between earth bags used to construct embankments. These voids occur because the earth bags are broken under the impact of seepage water. Usually, it is difficult to recognize these voids because they are different sizes and expand easily. Animal caves (i.e., mouse holes, badger caves, termite caves, etc.) are common hidden defects found in the conventional soil structures. Commonly, the diameter of these caves ranges from $40 \mathrm{~cm}$ to $60 \mathrm{~cm}$ and can even reach several metres at the maximum. The passages connecting different caves are unfixed and extend in all directions. These hidden caves may influence the overall safety of hydraulic structures and increase seepage failure occurrences.

In separations between a concrete slab and foundation caused by seepage failure, there are usually some liquid substances present, such as mud in the small voids between the slab and foundation. The dielectric constants of air and these liquid substances in the void are much smaller than the dielectric constants of the common solid underground media. Thus, if the radar waves meet a void, the energy of the reflected waves is increased, and a powerful reflection signature will appear in the radar image. Simultaneously, the signal energy detected by the radar will not decrease gradually with an increase in depth because common reflected waves and some obvious signatures will appear in the lower part of a penetration profile, which indicates the appearance of voids in these locations.

The working frequency of the GPR system is wide, typically covering the very high frequency (VHF) band, ultra-high frequency (UHF) band and mobile communication frequency band, which is affected by electronic equipment and live wires between power supplies and the antenna. However, the detection areas in hydraulic engineering are often large and open, which has many advantages in GPR application. An open field is beneficial to the transmission and reception of an electromagnetic wave, as interference with a reflected wave caused by surrounding buildings usually does not appear. If there is electronic equipment such as a radio and mobile phone near the 


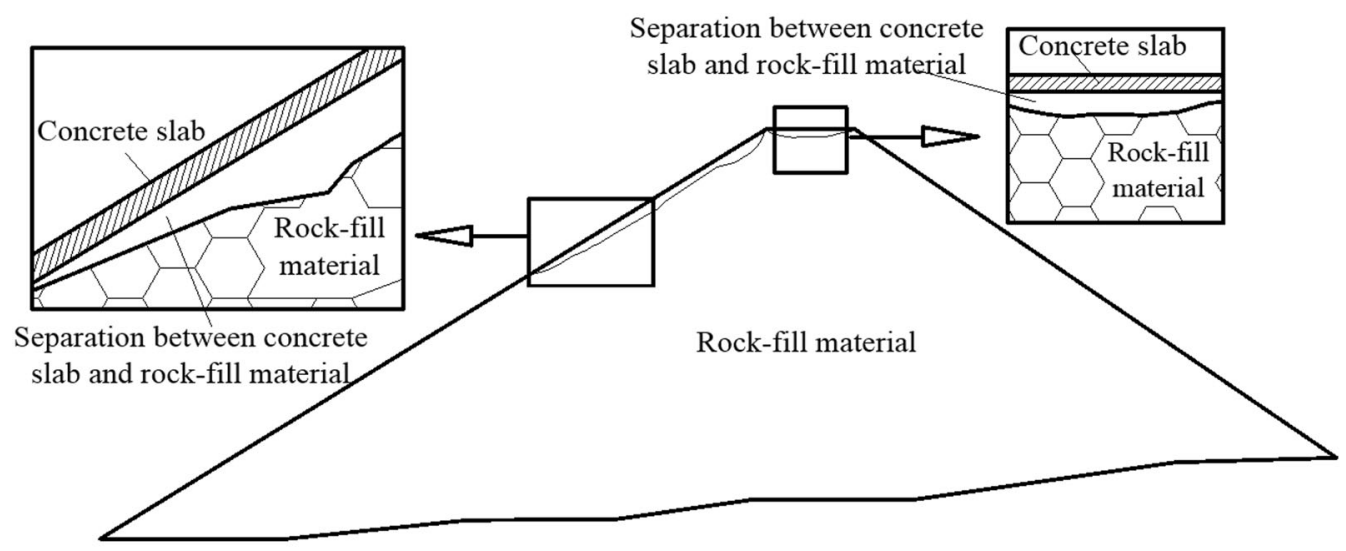

(a)


Void

(b)

Figure 1. Causes of the separation between concrete slab and foundation: (a) Separation caused by the uncoordinated deformation of an embankment and (b) a void under a sluice floor caused by seepage.

detection field, this equipment could be turned off during the detection process to avoid interferences. However, interference caused by live wires in the GPR system is unavoidable, and the persistent power supply of the live wires influences the penetration data in a continuous and stable manner.

In addition, the topography around hydraulic structures is typically very complex. We usually have to perform GPR detection on uneven ground to detect some key parts of the structure. As a result, the GPR detection antenna moves up and down during the penetration process, which may increase variation in the air direct coupled waves in the collected data and affect identification of target signatures.

Moreover, a wet detection field is very common problem in practical detection of hydraulic structures. For example, a sluice floor can be wet or even have stagnant water, and if it rained, the soil of the embankment structures will be wet and seepage may occur. It is well known that water is a medium with a high attenuation coefficient $\alpha$ (the attenuation coefficient $\alpha$ corresponding to seawater and distilled water can reach 1000-2000). When the media water content increases, its electrical conductivity $\sigma$ also increases. As a result, attenuation of the electromagnetic waves during the transmission process will increase. Therefore, the water content influence on underwater target detection is significant. In addition, at the interface between water and a solid medium, reflection and refraction of electromagnetic waves will occur. As waves are received by the radar system, the final collected data will show a significant difference compared to the actual data. With an increase in antenna frequency, reflection and refraction of the electromagnetic waves will strengthen, which will affect the penetration results of deep positions of the structure.

\section{Experimental method to assess the effects of different detection environments}

\subsection{Experimental models}

Two experimental models are designed to represent two kinds of hydraulic structures. Model A (see figure 2) is composed of a concrete slab in the upper and bottom clay structures. The concrete slab (size: $150 \mathrm{~cm} \times 100 \mathrm{~cm} \times$ $15 \mathrm{~cm})$ represents the sluice floor, and the clay structure (size: $170 \mathrm{~cm} \times 120 \mathrm{~cm} \times 70 \mathrm{~cm}$ ) represents the foundation. In the experiment, inflatable rubber balls, i.e., No. 1 football (diameter $13 \mathrm{~cm}$ ), No. 3 basketball (diameter 




(a)

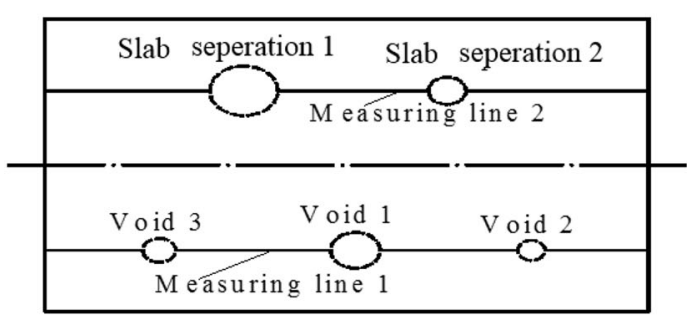

(b)

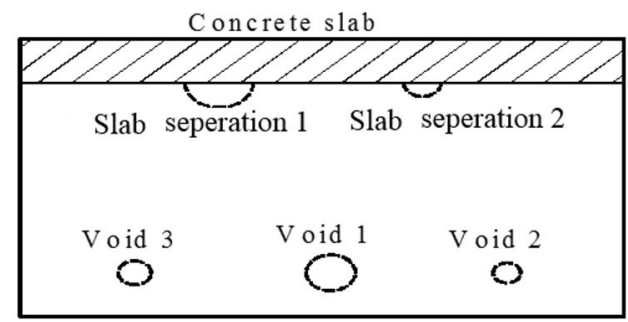

(c)

Figure 2. Model A, the simulated separation (between the concrete slab and foundation) and 3 voids in the foundation: (a) Operation in model A, (b) Plan view, and (c) Profile.

$17 \mathrm{~cm}$ ) and No. 5 football (diameter $21 \mathrm{~cm}$ ), are used to simulate three voids in the foundation. Since the relative dielectric constant of rubber $(\varepsilon=2)$ is similar to that of air $(\varepsilon=1)$, the interference caused by rubber can be largely reduced, and thus, the accuracy of the experiment is guaranteed. The separation between a concrete slab and foundation is simulated by digging under the slab at two locations in the foundation.

Model B with one measuring line constructed by earth bags is adopted to simulate the embankment, and some voids between bags are filled with soil. The size of model B is $180 \mathrm{~cm} \times 65 \mathrm{~cm} \times 120 \mathrm{~cm}$ (the size of each earth bag is $65 \mathrm{~cm} \times 45 \mathrm{~cm} \times 20 \mathrm{~cm}$ ) and the arrangement of the earth bags is shown in figure 3. In the experiments, some voids between earth bags are not filled with soil to simulate holes underground. Based on the relationship between the detection depth and radar resolution [14], the size and location of the voids are estimated and simulated, as shown in table 1 .

To assess the effects of the three environmental factors on the GPR detection results, the experiments are divided into reference experiments and comparison experiments. In the reference experiments, model $\mathrm{A}$ and model $\mathrm{B}$ are detected by the GPR with antennas of different frequencies, and corresponding reference data without interference of environmental factors is collected. For the comparison experiments, the two experimental models are modified to simulate the interference of the three environmental factors, and then, the detection is conducted using the modified models to collect comparison penetration data. The comparison experiments are divided into three types to assess the effects of different environmental factors (noise signal interference, uneven ground surface, and water interference). Then, the reference data and comparison data are compared to assess the effects of the environmental factors.

The SIR-20 GPR produced by LAUREL (US) is used for the detection. Combined with related references about GPR detection in similar structures and the operation instruction of the SIR-20 GPR, frequencies including $1 \mathrm{GHz}$, $1600 \mathrm{MHz}, 900 \mathrm{MHz}, 400 \mathrm{MHz}$ and $100 \mathrm{MHz}$ are selected for the GPR detection in the two experimental models. The detection result of the $1 \mathrm{GHz}$ frequency is similar to that of the $1600 \mathrm{MHz}$ frequency, and the detection result of the $900 \mathrm{MHz}$ frequency is similar to that of the $400 \mathrm{MHz}$ and $100 \mathrm{MHz}$ frequencies. Thus, frequencies of $100 \mathrm{MHz}$, $900 \mathrm{MHz}$ and $1600 \mathrm{MHz}$ with fine detection results are chosen for analysis in this paper, and the parameters of the GPR system are shown in table 2. The collected data are analysed using RADAN5 software and some MATLAB programmes that we developed.

\subsection{Experiments to assess the effects of uneven ground surface}

Simulation of the uneven ground surface of the practical detection field allows us to evaluate the uneven ground effects on the GPR detection data. In the experiment, model $\mathrm{A}$ and model $\mathrm{B}$ are modified, and the uneven ground 


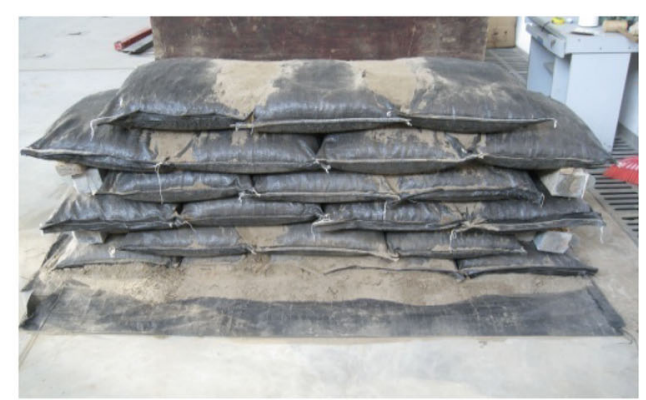

(a)

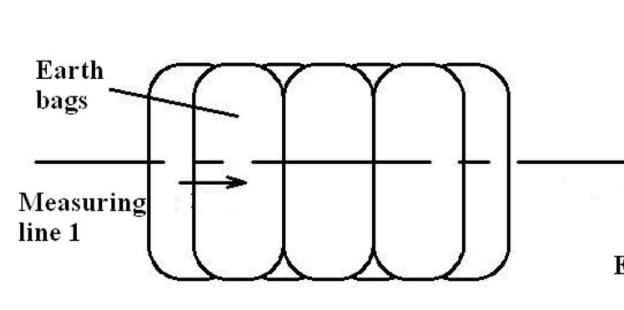

(b)

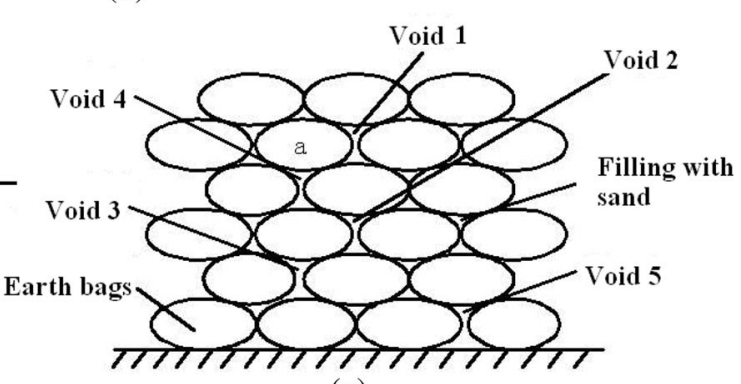

(c)

Figure 3. Model B and 5 simulated voids: (a) Appearance of model B, (b) Plan view, and (c) Profile.

Table 1. The size of the voids and slab separation in model $\mathrm{A}$ and model $\mathrm{B}$.

\begin{tabular}{lccccc}
\hline Model A & $\begin{array}{c}\text { Void } \\
1\end{array}$ & $\begin{array}{c}\text { Void } \\
2\end{array}$ & $\begin{array}{c}\text { Void } \\
3\end{array}$ & $\begin{array}{c}\text { Slab } \\
\text { separation } \\
1\end{array}$ & $\begin{array}{c}\text { Slab } \\
\text { separation }\end{array}$ \\
$\begin{array}{c}\text { Diameter } \\
(\mathrm{cm})\end{array}$ & 21 & 13 & 17 & 45 & 25 \\
Model B & $\begin{array}{c}\text { Void } \\
1\end{array}$ & $\begin{array}{c}\text { Void } \\
2\end{array}$ & $\begin{array}{c}\text { Void } \\
3\end{array}$ & Void 4 & Void 5 \\
$\begin{array}{c}\text { Diameter } \\
(\mathrm{cm})\end{array}$ & 6 & 3 & 2 & 1.5 & 1.0 \\
\hline
\end{tabular}

surface is simulated by covering the surface with some sand and gravel. The modified models are shown in figure 4. Then, the GPR with different frequency antennas are used for detection along the measuring lines of two test models, and the corresponding data are collected in different sections.
The two typical radar images related to the two models are shown in figure 5. The difference between the two models is only the ground surface and other parameters are the same. When GPR detection is performed on the uneven ground surface, the distance between the antenna and ground changes. Usually, the distance is less than $2 \mathrm{~cm}$ and varies by approximately $1 \mathrm{~cm}$. The random oscillation of the electromagnetic wave in the air layer between the antenna and ground will reduce the energy propagated downward. As a result, the amplitude of the signal in the lower part will decrease and reduce the identifiability of the structural defect signatures in the lower part of the radar image.

\subsection{Experiments to assess the effects of live wires}

Since the FM radio signal and GSM signal are in the range of the antenna frequency $(100 \mathrm{MHz}, 900 \mathrm{MHz}$ and $1600 \mathrm{MHz}$ ), we turn off nearby electronic equipment (i.e.,

Table 2. GPR parameters used in the experiment.

\begin{tabular}{llccccrr}
\hline $\begin{array}{l}\text { Antenna } \\
\text { frequency }\end{array}$ & $\begin{array}{c}\text { Acquisition } \\
\text { mode }\end{array}$ & $\begin{array}{c}\text { Sample } \\
\text { numbers }\end{array}$ & $\begin{array}{c}\text { Dielectric } \\
\text { constant } \varepsilon\end{array}$ & $\begin{array}{c}\text { Record } \\
\text { length (ns) }\end{array}$ & $\begin{array}{c}\text { High-pass } \\
\text { filtering (MHz) }\end{array}$ & $\begin{array}{c}\text { Low-pass } \\
\text { filtering (MHz) }\end{array}$ & $\begin{array}{c}\text { Gain setting } \\
\text { pattern }\end{array}$ \\
\hline $1600 \mathrm{MHz}$ & $\begin{array}{l}\text { Continuous } \\
\text { measurement }\end{array}$ & 512 & $8 / 10$ & $15 / 20$ & 100 & 800 & Automatically \\
$900 \mathrm{MHz}$ & $\begin{array}{l}\text { Continuous } \\
\text { measurement }\end{array}$ & 512 & 10 & $15 / 20$ & 100 & 800 & Automatically \\
$100 \mathrm{MHz}$ & $\begin{array}{l}\text { Continuous } \\
\text { measurement }\end{array}$ & 512 & 8 & 20 & 100 & 800 & Automatically \\
\hline
\end{tabular}




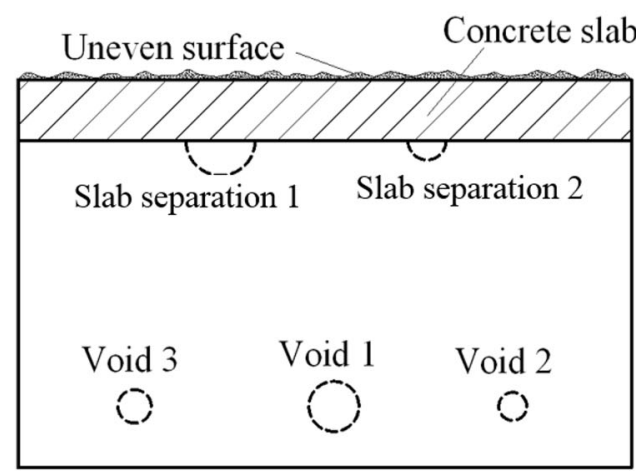

(a)

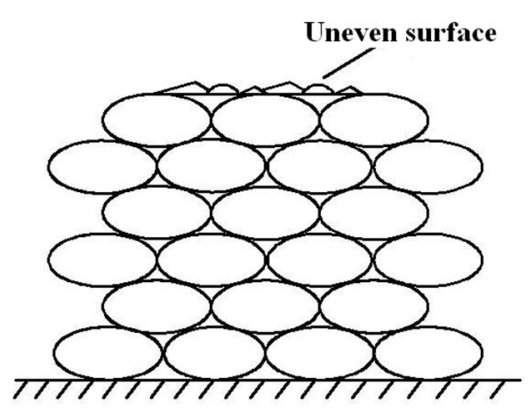

(c)

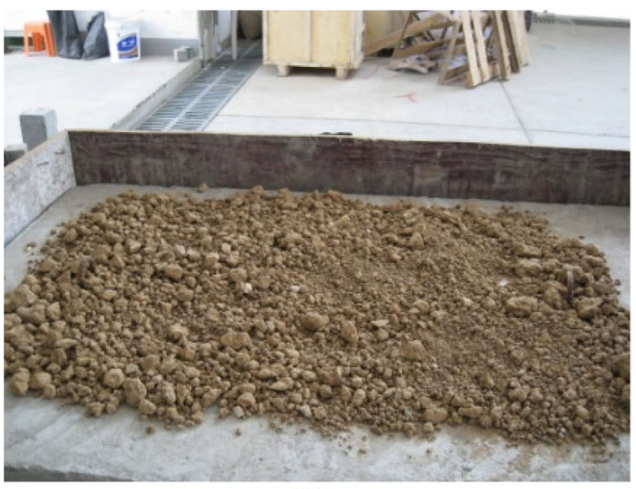

(b)

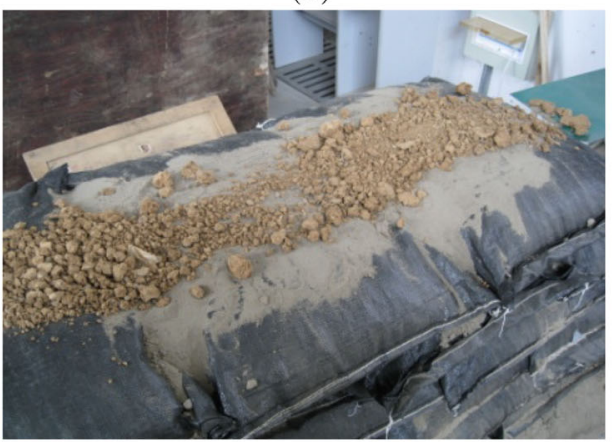

(d)

Figure 4. Modified model A and model B simulating the uneven penetration surface: (a) Profile of modified model A, (b) Photo of modified model A, (c) profile of modified model B, and (d) Photo of modified model B.

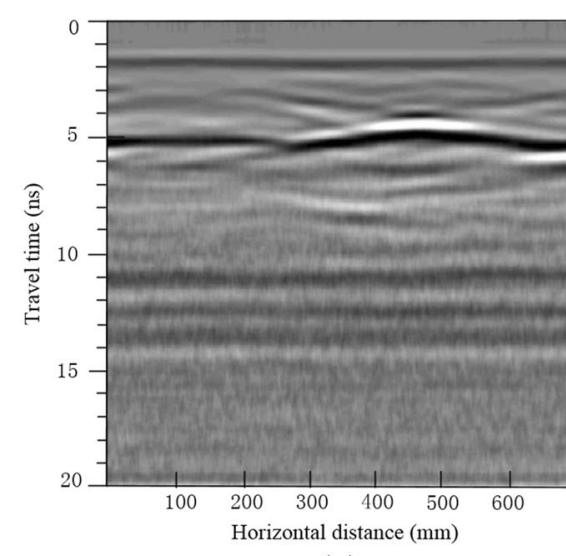

(a)



The oscillation amplitude of the signal in the low part is reduced

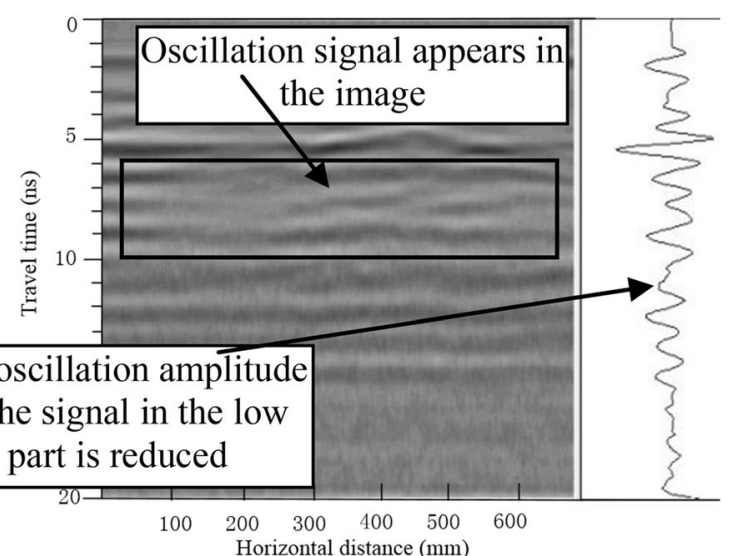

(b)

Figure 5. Typical GPR profile: (a) GPR profile when ground surface is even and (b) GPR profile when ground surface is uneven.

radio, TV and mobile phone) to avoid interferences. However, the noise signal produced by the live wires is inevitable because it is impossible for a GPR to transmit and receive waves without electricity. In this experiment, wires (power lines connecting to the GPR equipment) are placed next to the antenna, maintaining distances of $90 \mathrm{~cm}, 60 \mathrm{~cm}$ or $30 \mathrm{~cm}$. Then, along the measuring lines of model $\mathrm{A}$ and model B, the GPR detection profiles are obtained and these profiles are analysed to assess the interference of live wires.

Generally, live wires in GPR detection have the characteristic of continuous conduction time and small instantaneous power, and thus, the interference signal of live wire is continuous, stable and implicit. As seen in the detection profile in figure 6 , the signatures corresponding to wire 


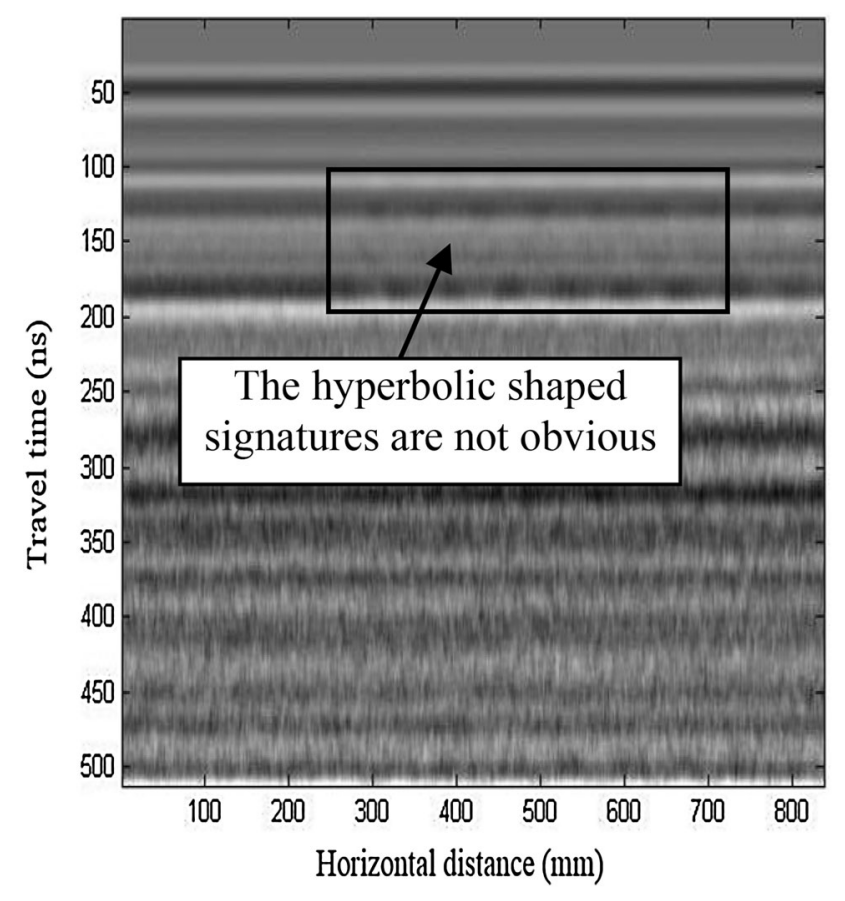

Figure 6. Typical GPR penetration profile under the interference of live wires.

interference have hyperbolic shapes. The signatures corresponding to the interference of live wires are implicit and continuous and the amplitude change of the waves in this part is not obvious, and thus, we must distinguish this change carefully.

\subsection{Experiments to assess the effects of water interference}

By performing GPR detection with water in different locations in the two experimental models, we attempt to set reasonable instrument parameters for the GPR system and determine the signatures corresponding to different wet conditions, which aids in identification of these signatures in the practical GPR detection.

In the experiment, some modifications are made to model $\mathrm{A}$ in the three different experimental cases:

Case a: the concrete slab and earth in the bottom of model are dry to simulate the dry environment.

Case b: the concrete slab is wet, and the earth is dry to simulate a sluice, where water has been completely drained recently.

Case c: the concrete slab and earth are both wet to simulate a sluice, where the water has not been completely drained.

The modification to model $\mathrm{B}$ is similar to that of model $\mathrm{A}$ and four different testing cases are studied:

Case 1: a completely dry model, which is consistent with the model used in the reference experiment;
Case 2: the surface of model B is wet, which is used to simulate an embankment after rain or other, similar wet conditions;

Case 3: there is water in a few gaps between different earth bags, which is used to simulate an embankment subject to local seepage; and

Case 4: a great amount of water exists in the gap between earth bags and water gradually permeates into the earth bags below, which is used to simulate an embankment where there is some water at the surface or leakage has occurred.

Modified experimental models A and B, which simulate different test cases with water, are shown in figure 7 . With these modified experimental models described above, GPR detection is implemented using antennas with different frequencies and detection data are collected along the measuring lines of different profiles in the models.

As the dielectric constant of water is much higher than that of air and earth bags, a strong reflection appears where two media are located. For the detection field with water, the electromagnetic waves are largely absorbed. The reflection and refraction of electromagnetic waves will occur at the interface between the water and solid medium, which are received by the radar system. As a result, a strong reflection may appear in the radar image. Three typical GPR detection profiles of model $\mathrm{B}$ corresponding to three different cases (case 2-case 4) are shown in figure 8. The figure shows that when the surface of the detection field has some water, since the effects of water are characterized by a strong reflection in the upper part of the image, the energy and frequency of the reflected waves are reduced. The signal waveform widens, and multiples appear in the lower part, which influences identification of other targets. When water exists at some positions inside the medium, a strong reflection of waves will occur in these positions. Since the area with water is not large, the distribution of these zones with strong reflections in the GPR profile appears as stripes. The presence of these zones with strong reflections caused by water will also affect the identification of voids below and strongly suppress the reflection waves of holes, especially for void signatures with a negative phase and large amplitude. In addition, when leakage occurs in the structure, the leak passage and surrounding media material are saturated, and the dielectric constant of these zones will increase. Consequently, the strength of reflected waves will increase, which leads to attenuation of the high frequency signal. The area will show a strong reflection in the GPR detection profiles.

\section{Results and discussions}

\subsection{Methods to reduce the interference caused by uneven ground}

It is known that uneven ground may disturb the GPR antenna while detecting underground media, which results 


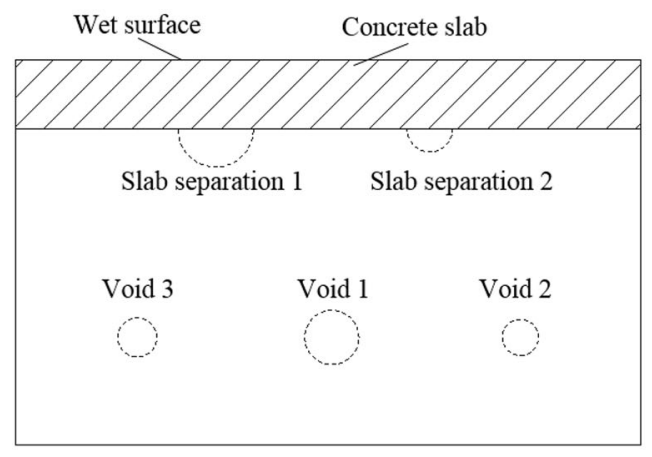

(a)

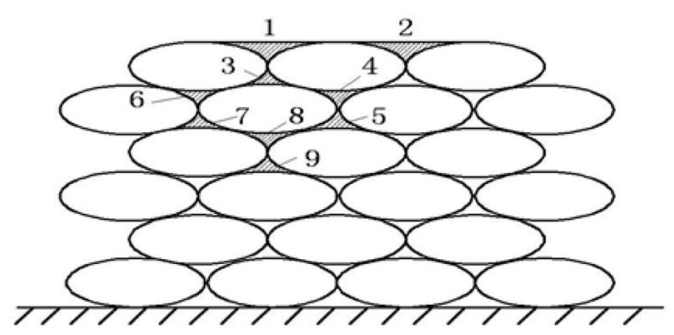

(c)

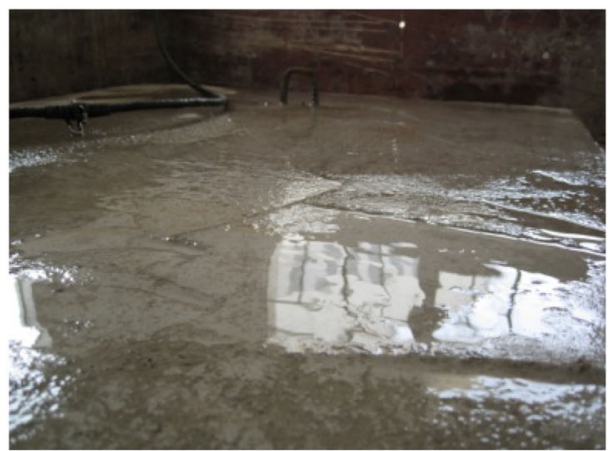

(b)

\begin{tabular}{|c|c|}
\hline Case & Position \\
\hline 1 & No water \\
\hline 2 & 1 and 2 \\
\hline 3 & 4 and 5 \\
\hline 4 & $1 \sim 9$ \\
\hline
\end{tabular}

Figure 7. Modified model A and model B: (a) vertical view of model A with a wet surface, (b) photo of model A with a wet surface, and (c) profile of model B and positions with water.

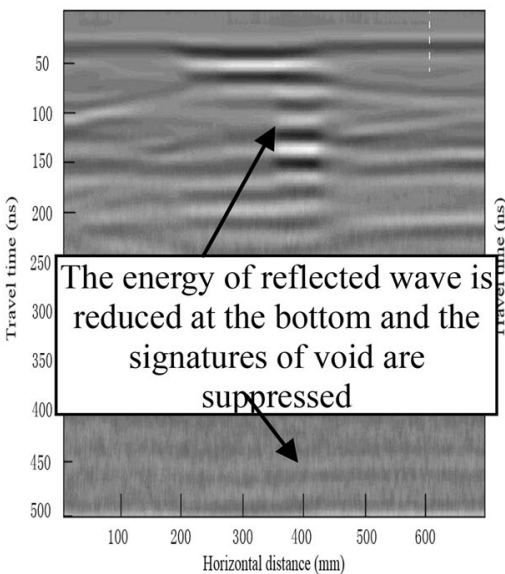

(a)

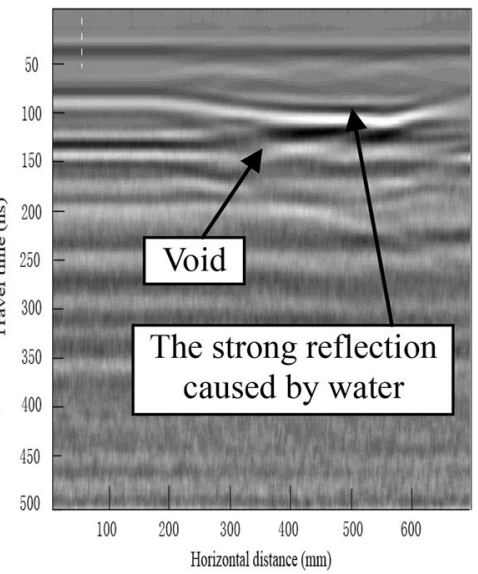

(b)



(c)

Figure 8. Typical GPR profile under the inference of water: (a) Water in the penetration surface, (b) water in a few gaps between earth bags, and (c) a large amount of water in the gap between earth bags.

in oscillation signatures. For this type of data, correlation of the points in the measuring line are embodied in the oscillation signatures. To solve the problem and highlight the oscillation signatures, the self-adaptive filter processing method is adopted since it has a high requirement for correlation. This aids in confirming and identifying the interference caused by uneven ground more effectively.

In the self-adaptive filter processing method, the data collected from even ground are equalized as the desired signal $d$. According to the principle of the adaptive transversal filter and the actual situation of the direct wave, a programme is compiled using MATLAB. Figure 9 shows the calculation procedures of the self-adaptive filter processing method [15].

In addition, due to the uneven ground surface, the direct wave from the ground surface changes randomly. As a result, in the signalling system received by the GPR, the direct wave appears earlier than the reflected waves, and the direct wave energy would concentrate in the earlier principle component. The principal component analysis (PCA) 


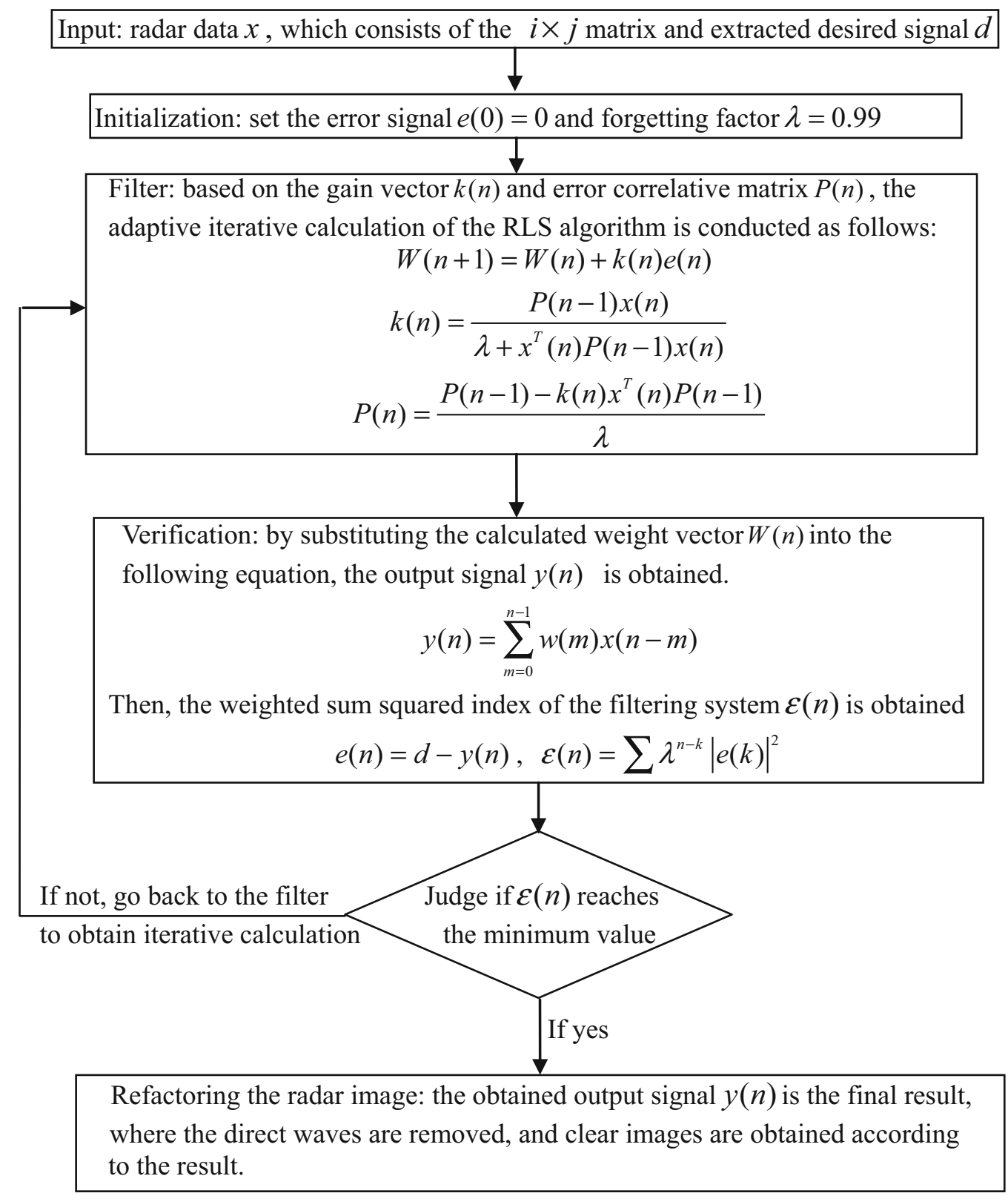

Figure 9. The calculation procedure of the self-adaptive filter processing method.

[16] method could be used to remove the direct wave interferences and recombine the effective signals based on comprehensively weighing the feature variables of the received signals, which has good effects during practical application.

The wavelet transform and two-dimensional filtering methods do not comprehensively weigh the data, which removes the interferences by eliminating partial fixed energy points. These methods do not remove the direct wave interferences entirely and may even remove the effective signals when the interference is weak. As a result, the subsequent interpretation would be affected.
In the principal component analysis method, the principle of the principal component analysis method and the actual direct wave situation are combined to compile a programme using MATLAB. The calculation procedures are shown in figure 10 [17].

In the data processing, the detection data is first adopted in the self-adaptive filter processing programme. Usually, from the image processed by self-adaptive filter, we can clearly see some oscillation signatures. These oscillation signatures indicate that the GPR antenna has been placed on an uneven ground surface during detection, which will greatly affect the identification of the underground target. 


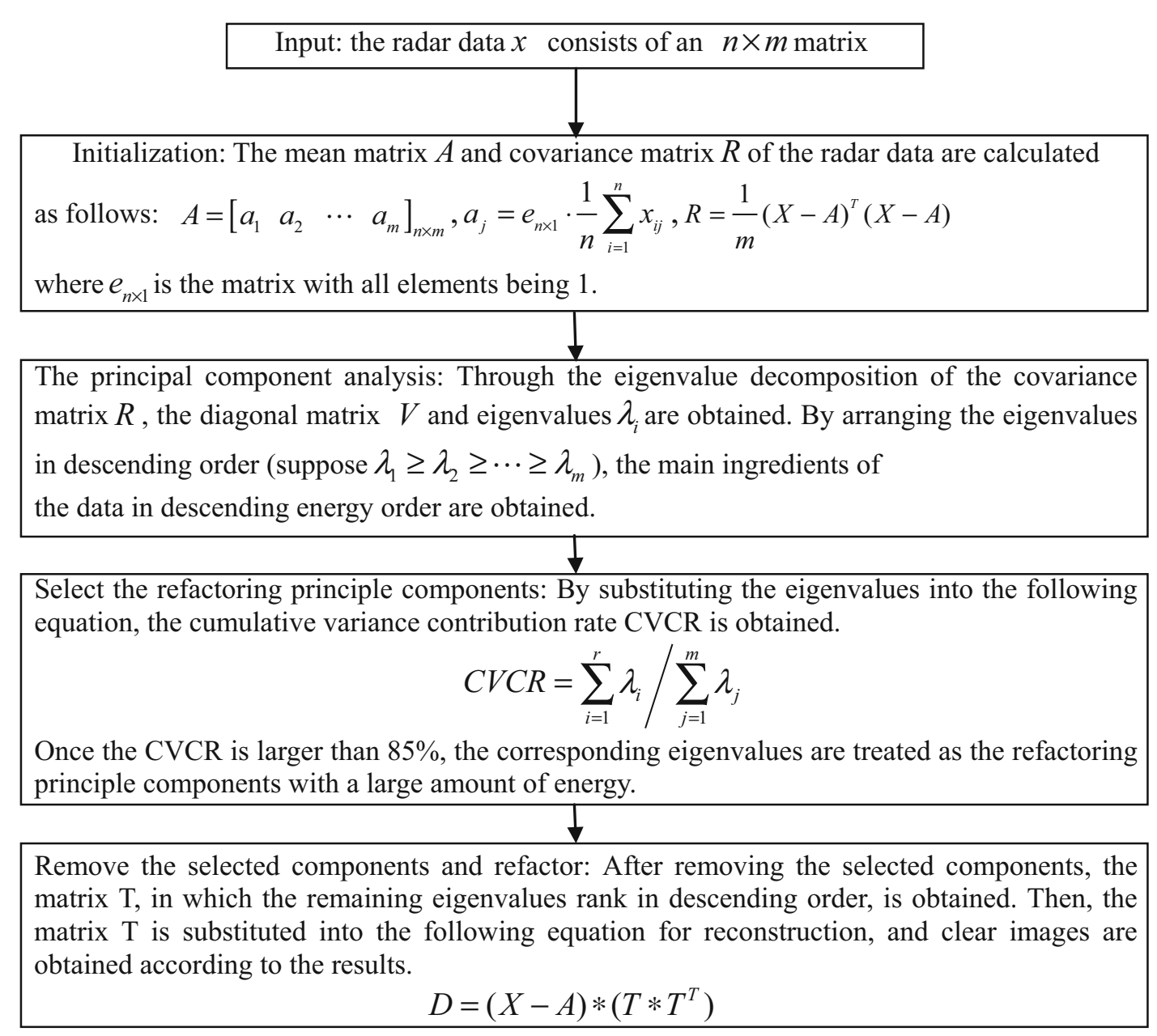

Figure 10. The calculation procedures of the principal component analysis method.

After using adaptive filtering, the processed data is adopted in the principal component analysis (PCA), and then, we obtain clear images, in which the influence of the uneven ground surface has been removed.

Figure 11 shows the GPR detection images processed using both methods. From this figure, we see that after the self-adaptive filtering process, the interference of direct waves are removed and the target signatures are highlighted. This method can largely maintain the primitiveness of the detection data, which facilitates the recognition and interpretation of detection data in the following work.

\subsection{Methods to reduce the interference oflive wires}

As shown in section 4.3, the interference signatures of the live wires are not usually very clear and can be difficult to identify directly from detection profiles. Therefore, before processing these images, we adopt methods to highlight these signatures to correctly determine the cause of interference in practical engineering, as the cause is unknown before the detection is conducted. Typically, the time- domain cancellation method is used to suppress the continuous and stable signatures caused by live wires [18]. In this method, among the detection data, two groups of data with a certain time gap are selected for the mutual reduction. Thus, the external noise signals $f_{\text {out }}(t)$ are obtained. Since the field environment of hydraulic engineering is large and the signal environment is stable, noise signals $f_{\text {out }}(t)$ can be treated as interference signals. By subtracting the formal detection data $f(t)$ from the noise signals $f_{\text {out }}(t)$, clear detection data $f_{w}(t)$ is obtained:

$$
f_{w}(t)=f(t)-f_{\text {out }}(t)
$$

To address the problems in this paper, GPR detection is first conducted with the transmitting antenna turned off and only the receiving antenna turned on. Then, the collected detection data can be taken as noise signals, which include the interference of live wires. Then, by subtracting the data from that of the normal GPR detection where the transmitting antenna and receiving antenna are both turned on, we obtain the final valid signal without interference from the external electromagnetic wave signal. In addition, 




(a)

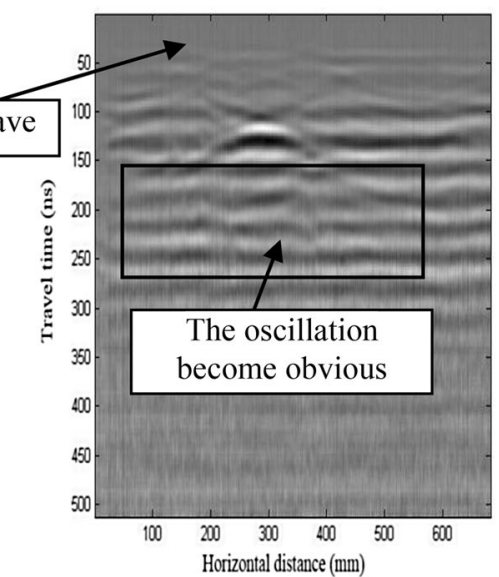

(b)

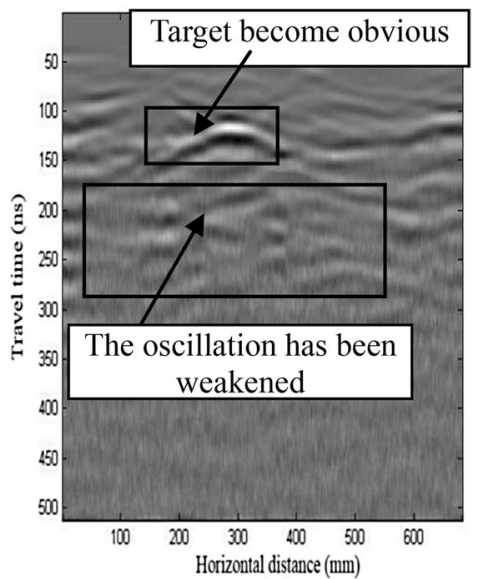

(c)

Figure 11. The image processing result: (a) original image, (b) after process using self-adaptive filtering, (c) after process using PCA.

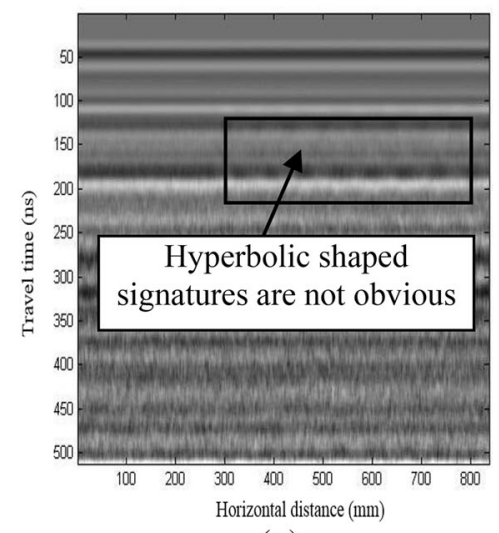

(a)

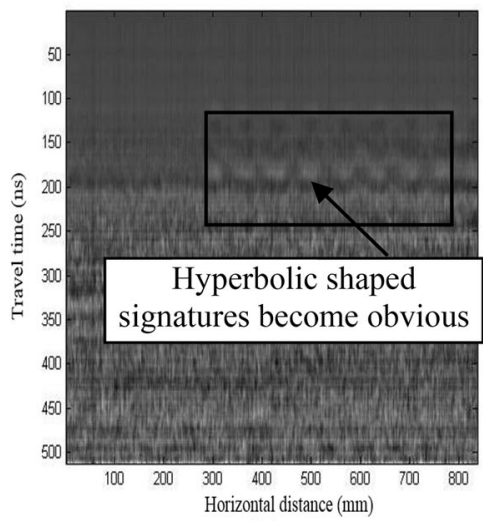

(b)

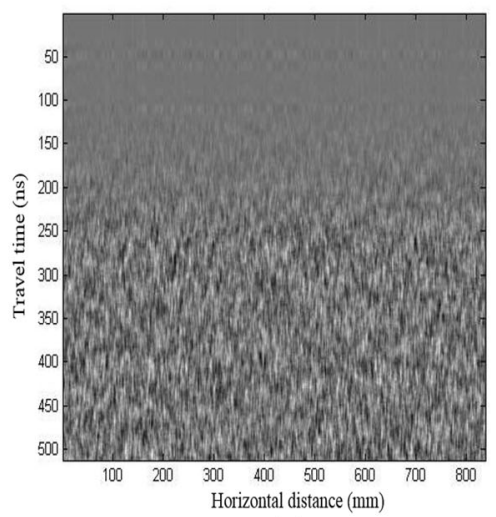

(c)

Figure 12. Typical GPR profile under the inference of live wires: (a) original image, (b) processed by adaptive filtering, and (c) processed by time-domain cancellation.

through the adaptive filtering method [19], the hyperbolic shaped signatures caused by the interference of live wires would be highlighted, and we can more easily determine the cause of the interference in the practical detection data and more reasonably interpret the data.

Figure 12 shows the processing result of a typical GPR profile using both methods. Through the adaptive filtering method process, hyperbolic shaped signatures become obvious and after processing the time-domain cancellation method, the hyperbolic shaped signatures caused by live wires are eliminated. Therefore, for the interference of continuous and stable noise signal, the processing result of both methods is satisfied.

\subsection{Methods to reduce the interference of water}

From the above test results, for the detection field with water, some zones with strong reflections appear in the GPR detection profiles and the detection target signatures are suppressed and may not be displayed in the image. To weaken the interference of water, we can take some measures during the process of data collection and adopt the deconvolution filtering method $[20,21]$ to process the GPR detection profiles.

Taking measures during the data collection process is the first and most important step to reduce the interference of water. Once the disturbance of water has been brought into the detection data, the effects of water cannot be removed and can only be weakened in the following process. Usually, before the implementation of GPR detection, we should carefully determine all visible and invisible locations with water within the detection field and attempt to clean it. The detection measuring lines should be arranged in a grid form and kept as far as possible away from the zones with water. If some zones with water cannot be avoided, detailed records of the zones should be taken, and if necessary, some additional measuring points would be added around these zones to better understand the area 


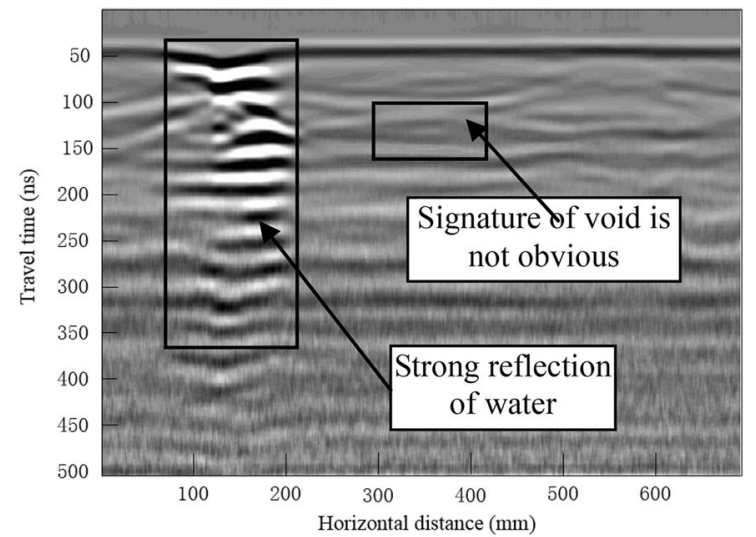

(a)

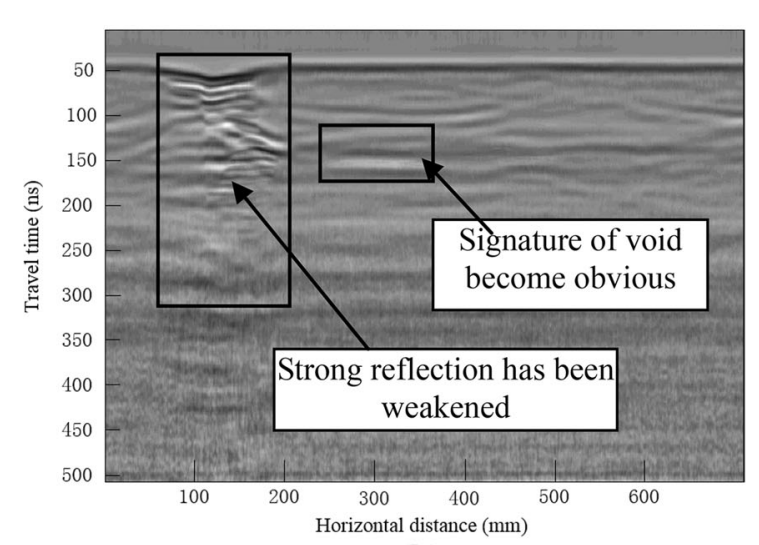

(b)

Figure 13. Image processing result using deconvolution: (a) untreated original image and (b) image after process using deconvolution.

details. For zones with water, before we begin the GPR detection system, the antenna is placed in the zones with water and the self-adaptive gain is used. Then, the gain of the GPR system will be adjusted automatically according to the water content of the detection profile to reduce the amplitude of the reflected waves and generate high quality radar images. In addition, since electromagnetic waves with different frequencies will be absorbed by water to different extents, the high and low frequency antennas are both used in the detection to complement each other. By comparing all GPR detection profiles with different antenna frequencies to each other, a strong reflection signature may be found in the same locations in all profiles. This means that there is some water around the location.

Sometimes, even after all the measures mentioned above have been taken, the interference of water in the detection data remains unavoidable. In this case, an image processing method should be adopted to further reduce the effects of water on the radar images. For a GPR detection profile with water interference, a strong reflection signature will appear where water exists. The deconvolution processing method, which substantially compresses such a strong reflection, can be used to concentrate the energy of a reflected wave on the position of its maximum energy. This method can widen the frequency bandwidth of the reflected waves, reduce the pulse dispersion and change the pulse waveform to obtain a higher resolution in the vertical direction.

In this study, deconvolution is conducted using RADAN5 software. For practical operations, some parameters including the operator length, unity gain, wavelength of output waves, prewhitening parameters, sampling time and so on have to be set by users. While the sampling time is determined by the collected data, the other parameters are set according to the practical detection situation. We have summarized the deconvolution process as follows.

Step 1: based on the extent of water interference on the GPR detection profiles, the operator length is set adaptively to change the range in which the reflected waves are compressed. Thus, the signatures caused by water interference are suppressed and the range affected by water is reduced. Generally, the greater the operator length, the better the effect of deconvolution filtering and a small operator length may generate a small, sharp pulse, resulting difficulty in identifying target signatures. Usually, for a limited data sequence, the value should be defined in a certain range, such as 20-60. Otherwise, it may increase the operation time and reduce the processing effect.

Step 2: if the water interference is very strong in the detection data and the normal target signal is suppressed, the wavelength of output waves and the prewhitening parameter can be adjusted to suppress the strong signal and strengthen the weak signal. The wavelength of output waves reflects the length of the delay time. The prewhitening parameter is used to modify the grey of the radar image. A large prewhitening parameter can increase the brightness of an image, so that the small signals, which are suppressed by the strong reflection of water, can be displayed.

Step 3: As mentioned above, GPR with automatic gain should be used for detection profiles involving water. Occasionally, the detection data may be obtained using GPR with a self-adaptive gain on detection profiles without water. In this case, an optimized GPR gain should be used through some mathematical method and the transmission power of the antenna is reduced or correspondingly increased.

Apply the image processing methods mentioned above to a GPR detection profile (see figure 13) obtained from model B with leakage water (test case 4). From figure 13, we can see that the refection caused by the seepage water is very strong, but the influence range is not too large. In this study, the operator length is set to 40 . Since the crest and trough of the reflected waves are relatively complete, we only use the self-adaptive gain without optimization. In addition, since the void signature is significantly suppressed, we can increase the identifiability of the image by 


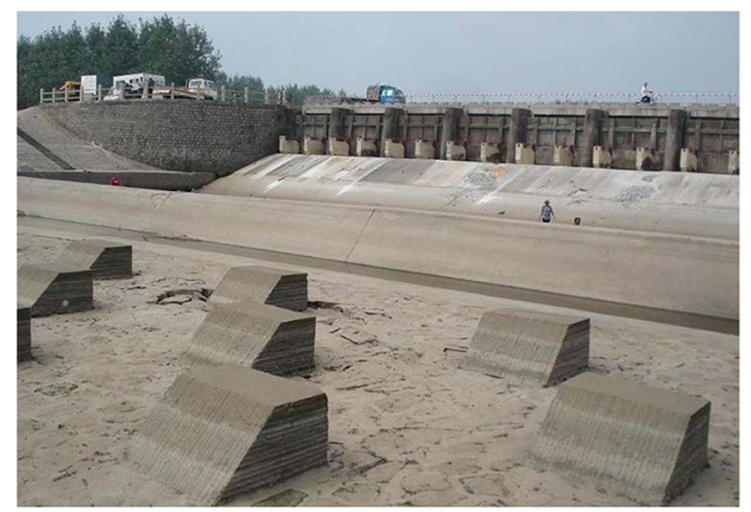

(a)

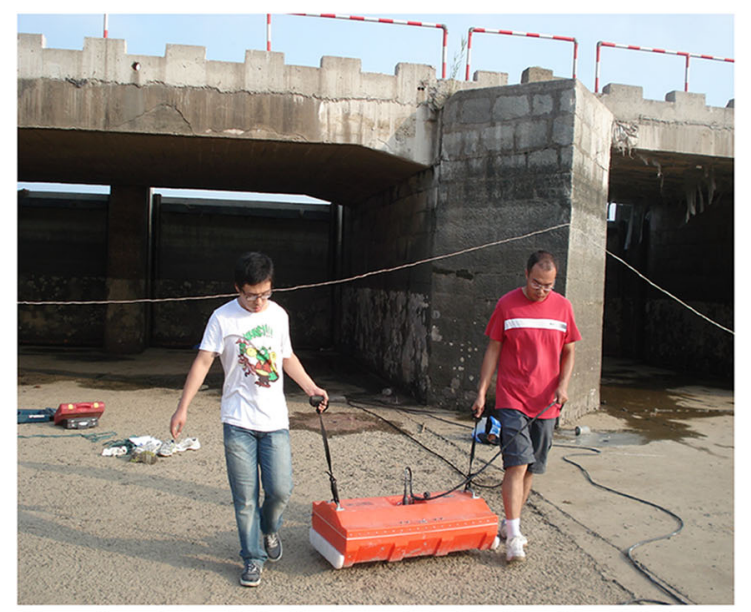

(c)

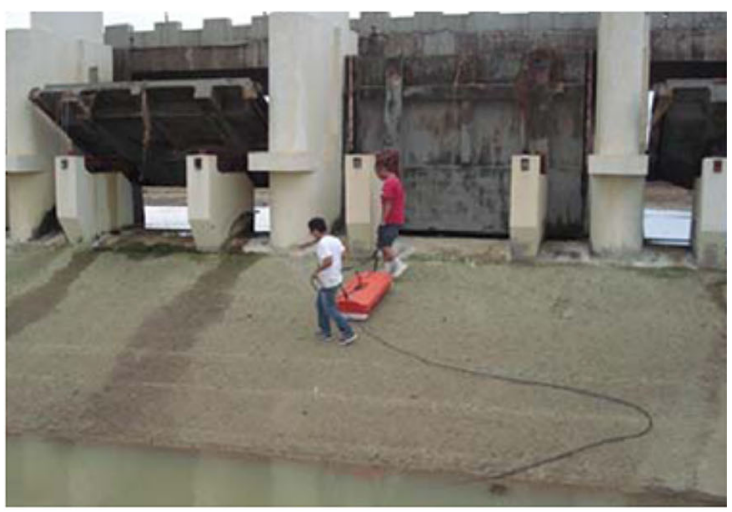

(b)

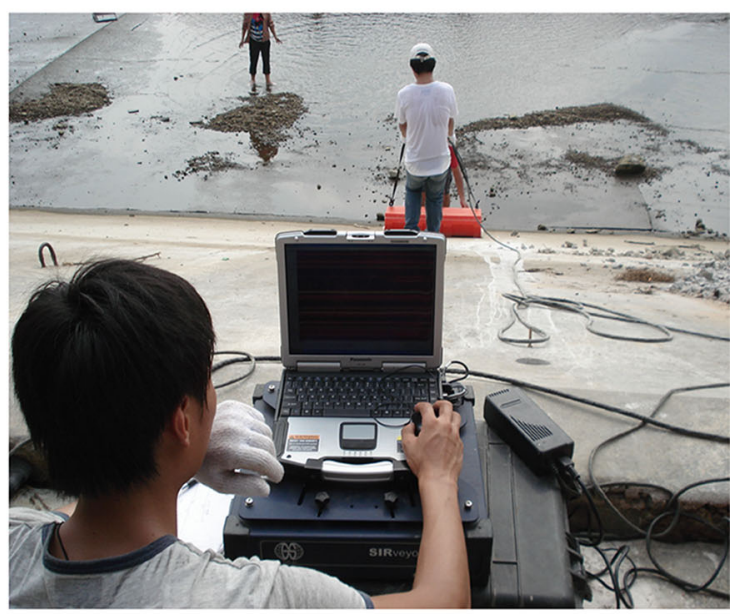

(d)

Figure 14. GPR detection field of the sluice: (a) sluice from a far view, (b) sluice gate nearby, (c) sluice chamber nearby, and (d) stilling basin nearby.

reducing the wavelength of output waves and increase the prewhitening parameter. After deconvolution filtering, radar waves under the interference of water are compressed, the influence range of water interference has been greatly reduced and each reflected wave caused by the water interference shrinks to the center of its maximum energy. Consequently, the strong reflection caused by water is suppressed. Simultaneously, the interference of other noise waves in the image is also restrained and the void signatures are highlighted. Therefore, the deconvolution processing method affects the processing of the radar image with water interference well.

\subsection{GPR detection for a sluice}

To verify the image features influenced by the three environmental factors and processing methods mentioned above, GPR detection is applied to one sluice, which is in the lower reach of the Xinshu River in China. This sluice functions as flood discharge, water level regulation, irrigation, reducing riverbed scouring, and so on. The sluice was built in 1957 with a total width of $169.6 \mathrm{~m}$ and 38 holes. Because the sluice has been running for a long time, aging and damages have already appeared. Through a preliminary examination in the field, we find water accumulation under the sluice floor and cracks on the sluice pier and bottom slabs. The detection environment is rather complex, which adds to the difficulty of GPR detection. Figure 14 illustrates the GPR detection field of the sluice.

Before the detection is conducted, the detection environment and electromagnetic properties of the detection medium are investigated. The interferences include phone signals, radio signals, live wires and metals, which have all been are recorded. Since the area of the detected sluice floor is large, criss-crossed measuring lines are adopted. A set of measuring lines perpendicular to the flow direction were arranged on the sluice floor before the survey, and the GPR detection is conducted along these measuring lines. For the area with abnormal detection results, measuring lines in the flow direction are arranged to have intensive detection, through which the locations and sizes of structural defects could be found.

Similarly, the SIR-20 GPR produced by LAUREL (US) is used for the detection. Some parameters of the GPR 
Table 3. Some GPR parameters used in the detection.

\begin{tabular}{llccccrc}
\hline $\begin{array}{l}\text { Antenna } \\
\text { frequency }(\mathrm{MHz})\end{array}$ & $\begin{array}{c}\text { Acquisition } \\
\text { mode }\end{array}$ & $\begin{array}{c}\text { Sample } \\
\text { numbers }\end{array}$ & $\begin{array}{c}\text { Dielectric } \\
\text { constant } \varepsilon\end{array}$ & $\begin{array}{c}\text { Record } \\
\text { length (ns) }\end{array}$ & $\begin{array}{c}\text { High-pass } \\
\text { filtering (MHz) }\end{array}$ & $\begin{array}{c}\text { Low-pass } \\
\text { filtering (MHz) }\end{array}$ & $\begin{array}{c}\text { Gain setting } \\
\text { pattern }\end{array}$ \\
\hline 100 & $\begin{array}{c}\text { Continuous } \\
\text { measurement }\end{array}$ & 512 & 10 & $60 / 250$ & 100 & 800 & Automatic \\
\hline
\end{tabular}

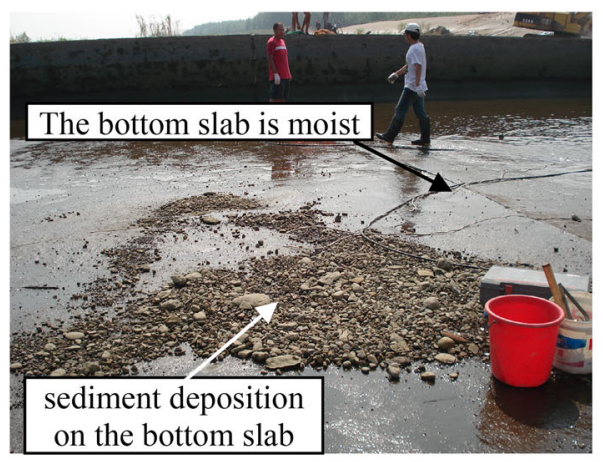

(a)



(b)

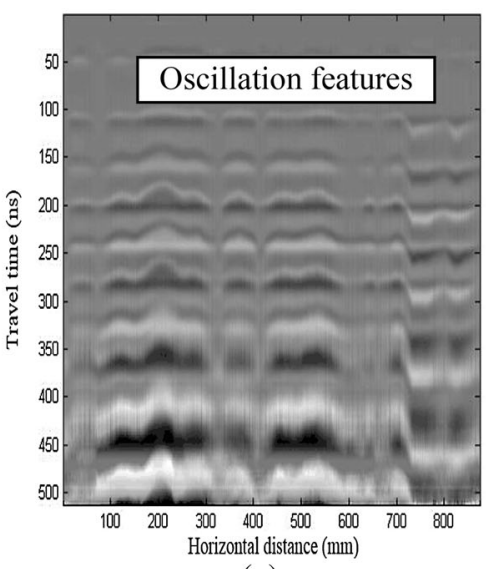

(c)

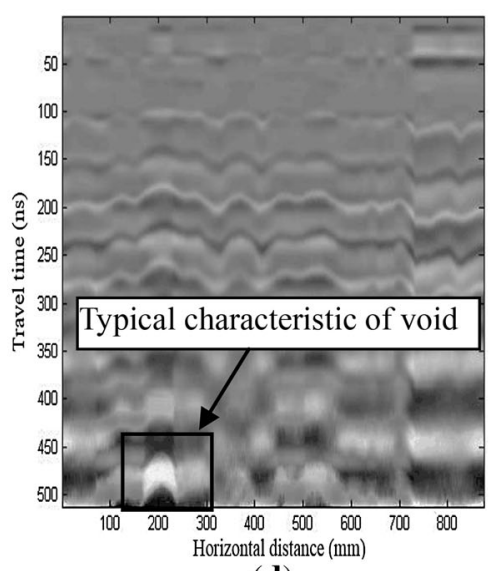

(d)

Figure 15. A practical example of the interference caused by an uneven ground surface. (a) The detection field of the stilling basin bottom slabs, (b) original image, (c) after processing the image using self-adaptive filtering, and (d) after processing using PCA.

system are shown in table 3. Among the detection results, the radar images influenced by the three environmental factors are selected for analysis.

4.4a The influence of the uneven ground surface: Based on an analysis of the radar image of the stilling basin bottom slabs shown in figure 15, the reflected waves are not steady and large amplitude signals appear in the lower part, which indicates the variation in the media in the detection area. Therefore, we primarily believe that there is a potential structural defect in this area. In the detection field, due to sediment deposition from running water, unevenly distributed sediment layers on the bottom slabs give rise to the uneven ground surface. In addition, since the water has been pumped for a few days, the bottom slabs and overflow surface are moist. This would affect the quality of the collected data.

Accordingly, the processing methods mentioned in section 4.1 are adopted. The detection data is first substituted into the self-adaptive filtering programme, and the obtained result is shown in figure 15c. Obviously, the oscillation signatures in the figure make it difficult to identify the image. Consequently, we believe that the interference is caused by the uneven ground surface. Then, by substituting the data into the principal component analysis method, the oscillation signatures in figure $15 \mathrm{~d}$ are weakened and the typical characteristic of a void can be clearly seen in the lower part of the image. Considering that there are no other 




(a)



(b)

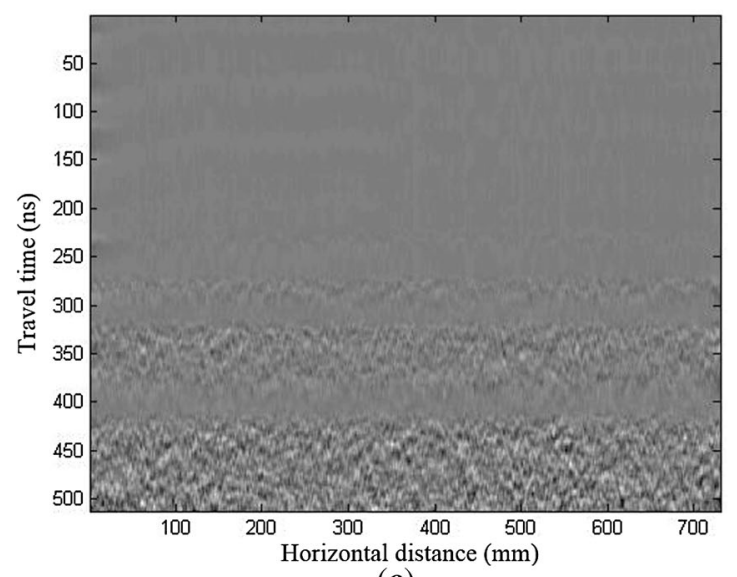

(c)

Figure 16. Practical example of the interference caused by live wires. (a) The detection field of the drop sill between stilling basins, (b) original image, and (c) after processing using the time-domain cancellation method.

typical characteristics of structural defects around, we believe a relatively small void may exist in the area. A void is found in the corresponding area after excavation, which verifies the accuracy of the detection. Thus, the processing methods corresponding to the uneven ground surface achieved a good effect in the practical application.

4.4b Influence of live wires: Continuous striatures can be seen in the radar image of the drop sill between stilling basins, as shown in figure 16, which interferes with data identification. Based on the characteristics of the interference caused by live wires in the case of a low frequency antenna mentioned in section 4.2, the continuous striatures are preliminary estimated to be the interference caused by the live wires. By checking the detection record in the field, live wires connected to the host of the SIR-20 GPR are present. The curly live wires can be seen in the hands of the detection personnel on the left in figure 16a. Since the live wires are within the interference distance, we conclude that the interference is caused by live wires.

Accordingly, the time-domain cancellation method is adopted to process the interference. The fifth channel data and the $105^{\text {th }}$ channel data among the previous detection data are selected to mutually reduce the external noise signal. Then, subtracting the detection data from the external noise signal, the interference is suppressed with the processed image as shown in figure 16c.

4.4c Influence of water: Similarly, GPR detection is applied to the area with stagnant water and sediment deposition along the bottom slabs of the stilling basins. Figure 17a shows two adopted measuring lines. Surely, the stagnant water on the bottom slabs shown in figure $17 \mathrm{~b}$ creates interference in the detection. For the collected data in the field shown in figure $17 \mathrm{c}$, a large, strong reflection area appears in the upper part of the image, the high frequency signals are weakened and the reflected waves in the lower part are suppressed.

Combining the processing methods mentioned in section 5.3, the deconvolution filtering method is adopted to process the detection data. To highlight the suppressed signals in the lower part, the gain parameter is increased and set to 3 , while other parameters used in the deconvolution filtering method are the same as those used in 


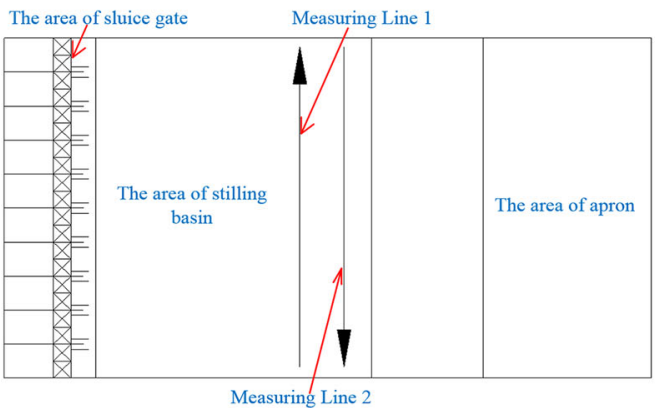

(a)

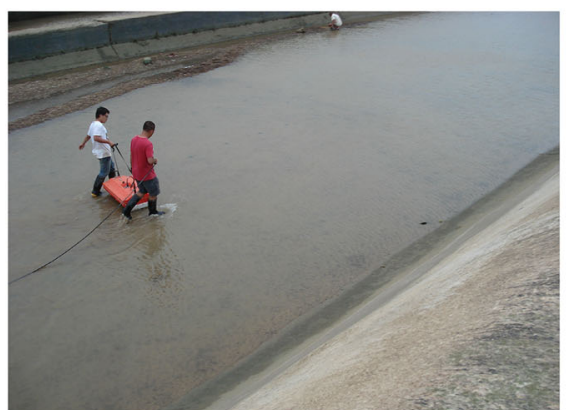

(b)

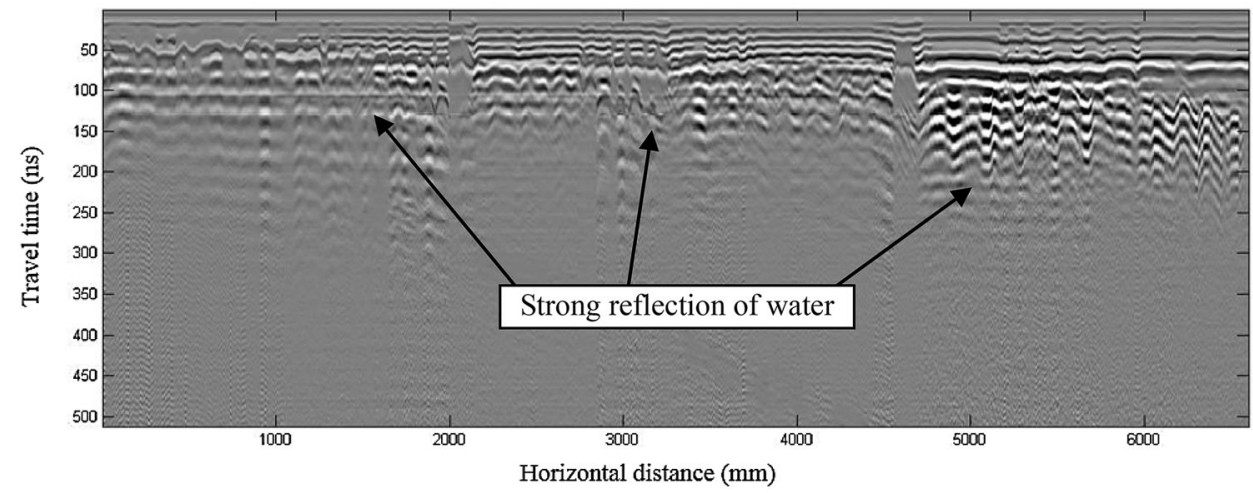

(c)

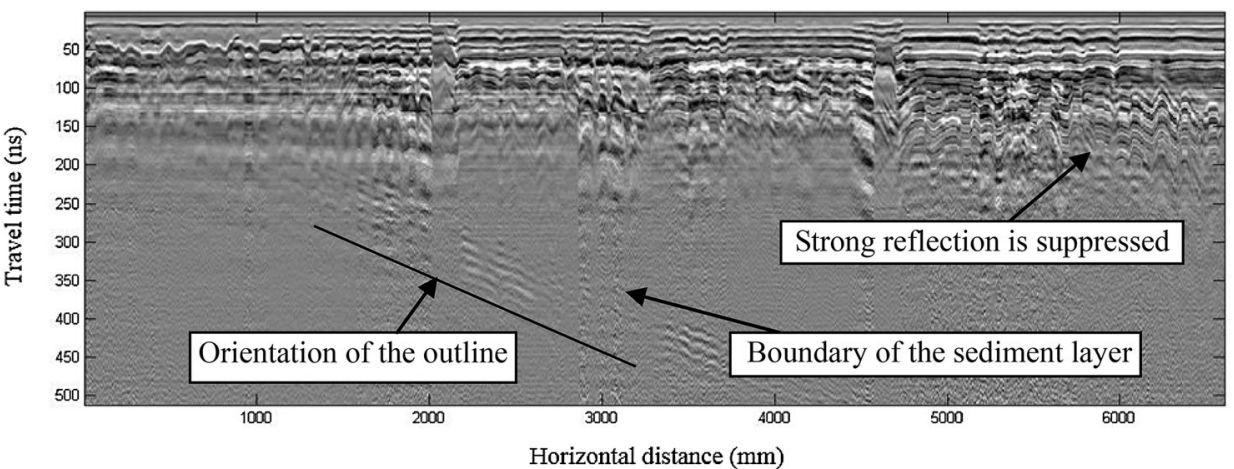

(d)

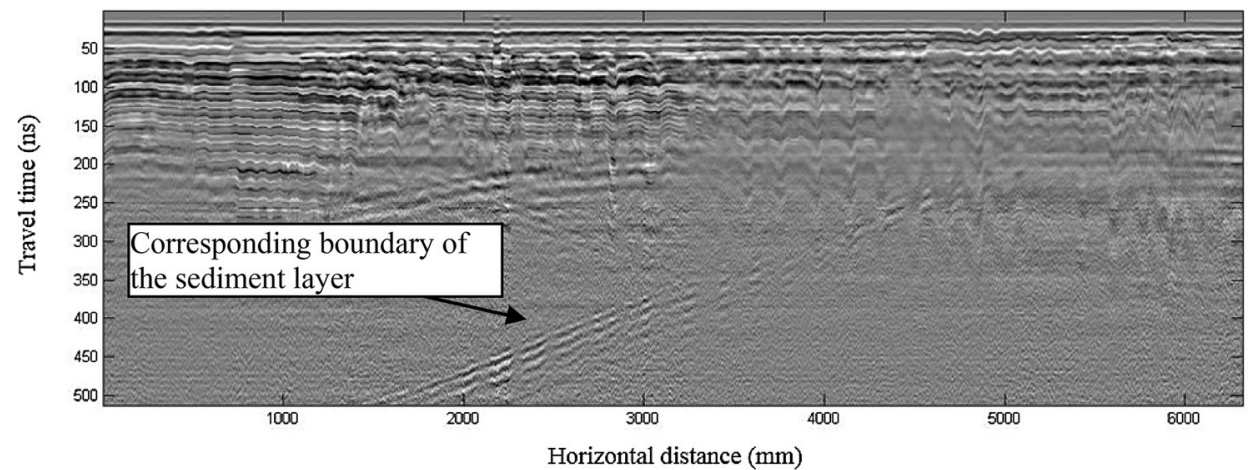

(e)

Figure 17. Practical example of the interference caused by water: (a) The measuring lines in the area with water, (b) the detection field, (c) original image in measuring line $1,(\mathbf{d})$ the processed image in measuring line 1 , and (e) the processed image in measuring line 1. 
Table 4. Image features and image processing methods corresponding to three environmental factors.

\begin{tabular}{|c|c|}
\hline $\begin{array}{l}\text { Environmental } \\
\text { factors }\end{array}$ & Image features \\
\hline $\begin{array}{l}\text { Clutter } \\
\text { interference } \\
\text { caused by live } \\
\text { wires }\end{array}$ & $\begin{array}{l}\text { (i) For the high frequency antenna, there are some } \\
\text { continuous hyperbolae in the image. } \\
\text { (ii) For low frequency antenna, there are some continuous } \\
\text { striatures. } \\
\text { (iii) The signatures are not obvious in the radar images or } \\
\text { cannot be seen. }\end{array}$ \\
\hline $\begin{array}{l}\text { Uneven } \\
\text { penetration } \\
\text { surface }\end{array}$ & $\begin{array}{l}\text { The amplitude of the signal in the lower part of the image is } \\
\text { weakened and the defect signatures are suppressed. }\end{array}$ \\
\hline \multicolumn{2}{|l|}{ Water interference } \\
\hline Wet surface & $\begin{array}{l}\text { (i) Some areas in the GPR penetration profile show strong } \\
\text { reflections. } \\
\text { (ii) The energy propagated down from the reflected waves } \\
\text { are reduced. }\end{array}$ \\
\hline & $\begin{array}{l}\text { (iii) The frequency of reflected waves is reduced, and the } \\
\text { waveform is broadened. }\end{array}$ \\
\hline Wet medium & $\begin{array}{l}\text { (i)-(iii) are similar to that listed above. } \\
\text { (iv) The distribution of strong reflection areas show some } \\
\text { stripes in the GPR detection profiles. }\end{array}$ \\
\hline Leakage & $\begin{array}{l}\text { (i)-(iii) are similar with that listed above. } \\
\text { (iv) The range of the strong reflection area is very large in } \\
\text { the GPR detection profiles and the strength of reflected } \\
\text { waves is great. }\end{array}$ \\
\hline
\end{tabular}

Processing methods

(i) During GPR penetration, the live wires of the antenna should be gathered by hand and kept a certain distance away from the antenna.

(ii) Use the adaptive filtering method to highlight the invisible influence signatures of noise waves.

(iii) Use the time-domain cancellation method to eliminate the noise waves.

(i) Optimize the measuring lines and try to level the uneven penetration surface before the GPR penetration is implemented.

(ii) Use the self-adaptive filtering method to highlight the unclear oscillation features caused by the uneven penetration surface.

(iii) Adopt the PCA method to eliminate the interference of water.

(i) Optimize the measuring lines to avoid positions with stagnant water.

(ii) Find all the positions with stagnant water in the field and try to clear the water before the GPR penetration is conducted.

(iii) For a penetration profile with water, the selfadaptive gain is used; otherwise, the gain of the antenna should be optimized.

(iv) Use the deconvolution filtering method to suppress the strong reflection of water. section 5.3. For the image processed after deconvolution shown in figure $17 \mathrm{~d}$, the strong reflection caused by water is suppressed and the boundary of the sediment layer in the lower part is highlighted. Moreover, corresponding to the boundary of the sediment layer in the processed image of measuring line 1 , the boundary of the sediment layer can be found in the processed image of measuring line 2, which further verifies the detection result accuracy. Therefore, the measures used to reduce the interference caused by water work well in the practical application.

\section{Conclusion}

For different environmental factors, the theory of interference is different in nature. As a result, the signatures of these interferences and the image processing methods also have significant differences. The GPR detection results from the two experimental models show us how each environmental factor affects the radar images and we obtain some typical GPR detection profiles with interference from each environmental factor. The measures used to reduce the interference caused by environmental factors make the images clearer. The GPR detection of a sluice verifies the validity of the experimental result and processing methods.

For practical engineering problems, these typical radar images can help us to determine the cause of the interference and distinguish the signatures of real structural defects. Correctly identifying the factors interfering with the detection data can aid us in choosing a reasonable image processing method.

To make the application of the above research results more convenient in practical engineering, we summarized in table 4 the signatures of radar images and image processing methods corresponding to the three kinds of environmental factors. In our future work, we intend to develop more effective interference reduction methods and apply these methods to practical engineering. 


\section{Acknowledgements}

This work is supported by National Key R\&D Program of China (Grant No. 2016YFC0401601), the National Natural Science Foundation of China (Grant Nos. 51579085, 41323001, 51139001, 51279052, 51379068, 51579083, 51579086, 51209077, 51479054), China State Key Laboratory of Hydrology-Water Resources and Hydraulic Engineering (Grant No. 20145028312), Jiangsu Province "333 High-Level Personnel Training Project" (Grant Nos. 2016-B1307101, 2017-B08037), National Key R\&D Program of China (Grant No. 2016YFC401601), Scientific innovation research of college graduated in Jiangsu Province (Grant No. KYZZ15_0140), the Fundamental Research Funds for the Central Universities (Grant Nos. 2015B32514, 2015B33314, 2016B04114),Huai' an Water Conservancy Academician Workstation, China State Key Laboratory of Hydrology-Water Resources and Hydraulic Engineering(Grant No. 20145028312), Project Funded by the Priority Academic Program Development of Jiangsu Higher Education Institutions (Grant No. YS11001), Jiangsu Basic Research Program (Grant No. BK20160872).

\section{References}

[1] Osamu Minemura, Noboru Sakata and Shigenori Yuyama 1998 Acoustic emission evaluation of an arch dam during construction cooling and grouting. Construction and Building Materials 12(6-7): 385-392 https://doi.org/10.1016/ S0950-0618(97)00082-2

[2] Zheng D J, Cheng L and Li X Q 2012 Face slab dispatch detection of concrete faced rock-fill dam. In: Proceedings of ASCE Earth and Space 2012 Conference, Pasadena, California, USA, pp. 858-866

[3] Loh C H and Wu T S 1996 Identification of Fei-Tsui arch dam from both ambient and seismic response data. Soil Dynamics and Earthquake Engineering 15(7): 465-483 https://doi.org/10.1016/0267-7261(96)00016-4

[4] Lal Mohit and Tiwari Rajiv 2018 Experimental identification of shaft misalignment in a turbo-generator system. SadhanaAcademy Proceedings in Engineering Science 43(5). https://doi.org/10.1007/s12046-018-0859-1

[5] Jol H M 2009 Ground Penetrating Radar Theory and Applications 51(3): 595-604. https://doi.org/10.1016/B9780-444-53348-7.00019-3

[6] Lu Yizhu, Song Wenlong, Lu Jingxuan, Su Zhicheng and Liu Hong 2017 Soil water measurement by ground penetrating radar and its scale features. South-to-North Water Transfers and Water Science \& Technology 15(2): 37-44. https://doi. org/10.13476/j.cnki.nsbdqk.2017.02.006

[7] Ghodoosi F, Bagchi A, Zayed T and Hosseini M R 2018 Method for developing and updating deterioration models for concrete bridge decks using GPR data. Automation in Construction 91: 133-141 https://doi.org/10.1016/j.autcon.2018. 03.014

[8] Sagnard F and Tarel J P 2016 Template-matching based detection of hyperbolas in ground-penetrating radargrams for buried utilities. Journal of Geophysics and Engineering 13(4): 491-504 https://doi.org/10.1088/1742-2132/13/4/491

[9] Liu L B and Xie X Y 2013 GPR for geotechnical engineering. Journal of Geophysics and Engineering 10(3) https://doi.org/10.1088/1742-2132/10/3/030201

[10] Li J, Zeng Z F, Slob E, Chen X and Liu F S 2014 Simulation of GPR passive interferometry using cross-correlation for LNAPL model monitoring application. Geophysical Journal International 199(3): 1919-1928 https://doi.org/10.1093/gji/ ggu367

[11] Wang G Q and He K S 2006 Detecting badger cave disease in embankment by ground penetrating radar. Rock and Soil Mechanics 27(5): 838-841

[12] Xue W, Zhu J C, Rong X, Huang Y J, Yang Y and Yu Y Y 2017 The analysis of ground penetrating radar signal based on generalized $\mathrm{S}$ transform with parameters optimization. Journal of Applied Geophysics 140: 75-83 https://doi.org/10. 1016/j.jappgeo.2017.03.016

[13] Zhang Baosen, Zhang Fangxiu, Liu Ziyang, Han Hongwei and Li Zhijun 2017 Field experiment study of the characteristics of GPR images of Yellow River ice. South-to-North Water Transfers and Water Science \& Technology 15(1): 121-125. https://doi.org/10.13476/j.cnki.nsbdqk.2017.01.020

[14] Zeng C, Chen C and Xu S F 2004 The application of ground penetrating radar in the highway road investigation. Chinese Journal of Engineering Geophisics 1(2): 130-135

[15] Haykin S 2002 Adaptive filter theory. Publishing House of Electronics Industry; Pearson 4(96): 469-490

[16] Kaplan G B, Icoglu O, Yoldemir A B and Sezgin M 2010 In: Proceedings of the International Conference on Ground Penetrating Radar. Lecce, Italy, pp. 1-6

[17] Gao Xiang 2011 Researches on signal processing and target recognition of ground penetrating radar for subsurface detection. Ph.D. Thesis, Ocean University of China

[18] Li Yi, Huang Chunlin and Lei Wentai 2006 The theory and application of Ground Penetration radar. Beijing, China: Science Press

[19] Jeng Y, Li Y W, Chen C S and Chien H Y 2009 Adaptive filtering of random noise in near-surface seismic and groundpenetrating radar data. Journal of Applied Geophysics 68(1): 36-46 https://doi.org/10.1016/j.jappgeo.2008.08.013

[20] Su M X, Li S C and Xue Y G 2010. GPR high resolution processing method based on the deconvolution. Journal of Zhejiang University (engineering edition). 44(6): 1201-1206

[21] Xia J H, Franseen E K, Miller R D and Weis T V 2004 Application of deterministic deconvolution of ground-penetrating radar data in a study of carbonate strata. Journal of Applied Geophysics 56(3): 213-229 https://doi.org/10.1016/ j.jappgeo.2004.07.003 